\title{
Unsteady MHD Poiseuille Flow between Two Infinite Parallel Plates in an Inclined Magnetic Field with Heat Transfer
}

\author{
A.S. Idowu and J.O. Olabode \\ Department of Mathematics, University of Ilorin, Ilorin, Nigeria
}

\begin{abstract}
Unsteady MHD Poiseuille flow between two infinite parallel porous plates in an inclined magnetic field with heat transfer has been studied. The pressure gradient is considered negligible. The lower plate alone is porous. The momentum and energy equations were solved by variable separable technique. The fluid velocity is expressed in terms of Hartmann number while temperature is in terms of Prandlt number. The effects of various magnetic inclinations to velocity and skin friction with various Prandlt number to temperature and Nulsselt number are discussed by graphs and tables.
\end{abstract}

Key Words: Magnetohydrodynamics(MHD) flow, Porous Plate, Heat Transfer.

\section{Introduction}

Magnetohydrodynamics (MHD) is concerned with the interaction of electrically conducting fluids and electromagnetic fields. When such fluid moves through a magnetic field, an electric field and consequently a current may be induced, and in turn the current interacts with the magnetic field to produce a body force on fluid. The production of this current has led to magnetohydrodynamics electrical power generator and the influence of a magnetic field viscous incompressible flow of electrically conducting fluid is of use in extrusion of plastics in the manufacture of Rayon and Nylon etc. MHD flow equations are combination of continuity equation and Navier-Stokes equation and Maxwell's equations.

Hannes Alfven (1942), a Swedish electrical engineer first initiated the study of MHD. Shercliff (1956) considered the steady motion of an electrically conducting fluid in pipes under transverse magnetic fields. Sparrow and Cess (1961) observed that the free convection heat transfer to liquid metals may be significantly affected by the presence of magnetic field. Drake (1965) considered flow in a channel due to periodic pressure gradient and solved the resulting equation by separation of variables methods. Singh and Ram (1978) studied Laminar flow of an electrically conducting fluid through a channel in the presence of a transverse magnetic field under the influence of a periodic pressure gradient and solved the resulting differential equation by the method of Laplace transform. More to this, Ram et al (1984) have analyzed Hall effects on heat and mass transfer flow through porous media. Soundelgekar and Abdulla Ali (1986) studied the flow of viscous incompressible electrically conducting fluid past an impulsively started infinite vertical isothermal plate. Singh (1993) considered steady MHD fluid flow between two parallel plates. John Mooney and Nick Stokes (1997) considered the numerical requirements for MHD flows with free surfaces. Raptis and Perdikis (1999) considered the effects of thermal radiation and free convection flow past a moving vertical plate. Al-Hadhrami (2003) discussed flow through horizontal channels of porous material and obtained velocity expressions in terms of the Reynolds number. Ganesh (2007) studied unsteady MHD Stokes flow of a viscous fluid between two parallel porous plates. Gbadeyan, Idowu et al (2010) studied the radiative effect on velocity, magnetic and temperature fields of a magnetohydrodynamic oscillatory flow past a limiting surface with variable suction. Stamenkovic et al (2010) investigates MHD flow of two immiscible and electrically conducting fluids between isothermal, insulated moving plates in the presence of applied electric and magnetic fields. He matched the solution at the interface and it was found that decrease in magnetic field inclination angle flattens out the velocity and temperature profiles. Rajput and Sahu (2011) studied the effect of a uniform transverse magnetic field on the unsteady transient free convection flow of an incompressible viscous electrically conducting fluid between two infinite vertical parallel porous plates with constant temperature and variable mass diffusion. Manyonge et al(2012) studied steady MHD Poiseuille flow between two infinite parallel porous plates in an inclined magnetic field and discover that high magnetic field strength decreases the velocity.

In this paper we considered one dimensional poiseuille flow of an electrically conducting fluid between two infinite parallel plates under the influence of inclined magnetic field with heat transfer. The pressure gradient is considered negligible and we assess the effect of magnetic field on the nature of flow if the lower plate is porousand the nature of heat transfer throughout the medium needed to be observed. The resulting differential equations were solved by analytical method and the solutions of velocity with skin friction are expressed in terms of Hartmann number while that of temperature and Nulsselt number were expressed in term of Prandlt number. 


\section{Mathematical Formulation}

A. magnetic field of field strength represented by the vector B at right angle to the flow of an electrically conducting fluid moving with velocity $\mathrm{V}$ was introduced. Here, an electric field vector denoted by $\mathrm{E}$ is induced at right angle to both $\mathrm{V}$ and $\mathrm{B}$ because of their interaction.

We assume that the conducting fluid exhibits adiabatic flow in spite of magnetic field, then we denote the electrical conductivity of the fluid by a scalar $\sigma$.

Lorentz force comes in place because the conducting fluid cuts the lines of the magnetic field in electric generator. This vector $\mathrm{F}$ is parallel to $\mathrm{V}$ but in opposite direction but is perpendicular to the plane of both $\mathrm{J}$ and

B. Laminar flow through a channel under uniform transverse magnetic field is important because of the use of MHD generator, MHD pump and electromagnetic flow meter.

Here, we consider an electrically conducting, viscous, unsteady, incompressible fluid moving between two infinite parallel plates both kept at a constant distance $2 \mathrm{~h}$. Both plates of the channel are fixed with no motion confirming plane poiseuille flow.

The equations of motion are the continuity equation

$$
\nabla \cdot V=0
$$

And the Navier-Stokes equation

$$
\rho\left[\left(\frac{\partial}{\partial t}+V \cdot \nabla\right)\right] V=f_{B}-\nabla P+\mu \nabla^{2} V
$$

Where $\rho$ is the fluid density, $f_{B}$ is the body force per unit mass of the fluid, $\mu$ is the fluid viscosity and $\mathrm{P}$ is the pressure acting on the fluid. If one dimensional flow is assumed, so that we choose the axis of the channel formed by the two plates as the $\mathrm{x}$-axis and assume that flow is in this direction. Observed that $\bar{u}, \bar{v}$ and $\bar{w}$ are the velocity components in $\bar{x}, \bar{y}$ and $\bar{z}$ directions respectively and bars denote dimensionless quantities, then this implies $\bar{v}=\bar{w}=0$ and $\bar{u} \neq 0$, then the continuity equation is satisfied.

From this we infer that $\bar{u}$ is independent of $\bar{x}$ and this will make $[(\mathrm{V} . \nabla) \mathrm{V}]$ in the Navier-stokes equation to vanish. The body force $f_{B}$ is neglected and replace with Lorentz force and from the assumption that the flow is one dimensional, it means that the governing equation for this flow is

$$
\frac{\partial \bar{u}}{\partial \bar{t}}=-\frac{1}{\rho} \frac{\partial \bar{p}}{\partial \bar{x}}+v \frac{\partial^{2} \bar{u}}{\partial \bar{y}^{2}}+\frac{F_{x}}{\rho}
$$

Where $v=\frac{\mu}{\rho}$ is the kinematics viscosity and $F_{x}$ is the component of the magnetic force in the direction of x-axis. Assuming unidirectional flow so that $\bar{v}=\bar{w}=0$ and $B_{x}=B_{z}=0$ since magnetic field is along y-direction so that $V=i \bar{u}$ and $B=B_{0 j}$ where $B_{0}$ is the magnetic field strength component. Now,

$F_{x}=\sigma\left[\left(i \bar{u} \times j B_{0}\right)\right] \times \mathrm{j} B_{0}(4)$

So that we have

$\frac{F_{X}}{\rho}=-\frac{\sigma}{\rho} B_{0}^{2} \bar{u}(5)$

Then (3) becomes

$\frac{\partial \bar{u}}{\partial \bar{t}}=-\frac{1}{\rho} \frac{\partial \bar{p}}{\partial \bar{x}}+v \frac{\partial^{2} \bar{u}}{\partial \bar{y}^{2}}-\frac{\sigma}{\rho} B_{0}^{2} \bar{u}(6)$

From (6), when angle of inclination is introduced, we have

$$
\frac{\partial \bar{u}}{\partial \bar{t}}=-\frac{1}{\rho} \frac{\partial \bar{p}}{\partial \bar{x}}+v \frac{\partial^{2} \bar{u}}{\partial \bar{y}^{2}}-\frac{\sigma}{\rho} B_{0}^{2} \bar{u} \sin ^{2}(\alpha)
$$

Where $\alpha$ is the angle between $\mathrm{V}$ and $\mathrm{B}$. Equation (7) is general in the sense that both field can be assessed at any angle $\alpha$ for $0 \leq \alpha \leq \pi$ and it is solved subject to boundary conditions $u(-1, t)=0$, at $t=0$ and $u(1, t)=1$ at $t=1$. Let $\mathrm{L}$ be the characteristics length,non-dimensionless quantitiesare givenas follows

$\bar{x}=x L, \bar{y}=y L, \bar{p}=p \rho \frac{v^{2}}{L^{2}}, \bar{u}=\frac{u v}{L}, \bar{t}=\frac{t L^{2}}{v}(8)$

We put (8) into (7) to get

$\frac{\partial u}{\partial t}=-\frac{\partial p}{\partial x}+\frac{\partial^{2} u}{\partial y^{2}}-\frac{\sigma L^{2} B_{0}^{2}}{\mu} \sin ^{2}(\alpha) u(9)$

And (9) becomes

$\frac{\partial u}{\partial t}=-\frac{\partial p}{\partial x}+\frac{\partial^{2} u}{\partial y^{2}}-M^{2} u(10)$

Where $\mathrm{M}=M^{*} \sin \alpha$ and $M^{*}=\mathrm{L} B_{0} \sqrt{\frac{\sigma}{\mu}}=\mathrm{Ha}$ and Ha is the Hartmann number given by $H a^{2}=\frac{\sigma L^{2} B_{0}^{2}}{\mu}$. We assume that the rate of $\frac{\partial p}{\partial x}$ is negligible, thus it is neglected and (10) becomes

$\frac{\partial u}{\partial t}=\frac{\partial^{2} u}{\partial y^{2}}-M^{2} u(11)$ 
The dimensional boundary condition is chosen as

$$
\begin{gathered}
\bar{t}=0, \quad \bar{u}(y, t)=0 \\
\bar{t}>0, \quad \bar{u}(-L, \bar{t})=0
\end{gathered}
$$

$\bar{u}(L, \bar{t})=\frac{v}{L}$

the solution of (11) under the non-dimensional boundary condition

$$
\begin{aligned}
t=0, & u(y, t)=0 \\
t>0, & u(-L, t)=0 \\
u(L, t)=1 &
\end{aligned}
$$

is given as

$u(y, t)=e^{-\lambda^{2} t}\left[\frac{e^{-\xi y}-e^{\xi(y+2)}}{e^{-\lambda^{2}}\left(e^{-\xi}-e^{3 \xi}\right)}\right](14)$

Where $\xi=\sqrt{M^{2}-\lambda^{2}}$

\section{MHD Fluid Flow between Two Infinite ParallelPorous Plates}

Because of the porosity of the lower plate, the characteristic velocity $v_{0}$ is taken as a constant so as to maintain the same pattern of flow against suction and injection of the fluid in which it is moving perpendicular to the fluid flow. The origin is taken at the centre of the channel and $\mathrm{x}, \mathrm{y}$ coordinate axes are parallel and perpendicular to the channel walls respectively. The governing equation is as follow

$\rho \frac{\partial \bar{u}}{\partial t}=-v_{0} \frac{\partial \bar{u}}{\partial \bar{y}}-\frac{\partial \bar{p}}{\partial \bar{x}}+\mu \frac{\partial^{2} \bar{u}}{\partial \bar{y}^{2}}-\frac{\sigma}{\rho} B_{0}^{2} \bar{u} \sin ^{2}(\alpha)(15)$

The dimensional form of (15) using (8) is

$\frac{\partial u}{\partial t}=-\frac{R_{e}}{\rho} \frac{\partial u}{\partial y}-\frac{\partial p}{\partial x}+\frac{\partial^{2} u}{\partial y^{2}}-M^{2} u(16)$

Where $\mathrm{M}=M^{*} \sin \alpha$ and $M^{*}=\mathrm{L} B_{0} \sqrt{\frac{\sigma}{\mu}}=\mathrm{Ha}$ and Ha is the Hartmann number given by $H a^{2}=\frac{\sigma L^{2} B_{0}^{2}}{\mu}$. We assume that the rate of $\frac{\partial p}{\partial x}$ is so negligible such that it can be neglected and (16) becomes,

$\frac{\partial u}{\partial t}=-\frac{R_{e}}{\rho} \frac{\partial u}{\partial y}+\frac{\partial^{2} u}{\partial y^{2}}-M^{2} u$

When (21) is solved, our solution shall be

$u(y, t)=\left[e^{\frac{A}{2} y-\lambda^{2} t}\right]\left\{\frac{e^{\eta y}-e^{-(y+2) \eta}}{e^{A / 2}-\lambda^{2}\left(e^{\eta}-e^{-3 \eta}\right)}\right\}$

where $A=\frac{R_{e}}{\rho}, \eta=\sqrt{\frac{A^{2}}{4}+M^{2}-\lambda^{2}}, M=M^{*} \sin \alpha, M^{*}=L B_{0} \sqrt{\frac{\sigma}{\mu}}$. Flow velocity for Hartmann numbers Ha $=$ $0.1,0.2,0.3,0.4$ and angle of inclinations $\alpha=15^{\circ}, \alpha=30^{\circ}, \alpha=60^{\circ}$ are depicted in the graph

\section{Effect of Temperature on the Fluid}

If the flow is assumed incompressible and the viscosity heating is ignored, the governing equation of heat is given as

$\frac{\partial \bar{T}}{\partial \bar{t}}=\frac{k}{\rho c_{p}} \frac{\partial^{2} \bar{T}}{\partial \bar{y}^{2}}$

Where $\mathrm{k}$ is the thermal conductivity of the fluid, $\rho$ is the density, $c_{p}$ is the specific heat at constant pressure and $\bar{T}$ the temperature. With the following dimensionless parameter:

So (19) now becomes

$$
P_{r}=\frac{\mu c_{p}}{k}, \theta=\frac{\bar{T}-\bar{T}_{\infty}}{\bar{T}_{w}-\bar{T}_{\infty}}, t=\frac{v \bar{t}}{L^{2}}
$$

$$
P_{r} \frac{\partial \theta}{\partial t}=\frac{\partial^{2} \theta}{\partial y^{2}}
$$

And the solution of (21) under the boundary condition $\theta(-1, t)=0$ at $t=0$ and $\theta(1, t)=1$

Where $\mathrm{A}=\frac{\lambda^{2}}{P_{r}}$

$$
\theta(y, t)=\frac{e^{A(1-t)}}{2}\left[\frac{\cos \lambda y}{\cos \lambda}+\frac{\sin \lambda y}{\sin \lambda}\right]
$$

\section{Skin Friction}

When both plates are non-porous

$\frac{\partial u}{\partial y}=-\xi e^{-\lambda^{2} t}\left\{\frac{e^{-\xi y}+e^{\xi(y+2)}}{e^{-\lambda^{2}}\left(e^{-\xi}-e^{3 \xi}\right)}\right\}$ 
Whenlower plate is considered porous

$$
\left[\frac{\partial u}{\partial y}\right]_{y=0}=\tau=-\xi e^{-\lambda^{2} t}\left[\frac{1+e^{2 \xi}}{e^{-\lambda^{2}}\left(e^{-\xi}-e^{3 \xi}\right)}\right]
$$

$$
\begin{aligned}
\frac{\partial u}{\partial y}=e^{-t \lambda^{2}}\left\{\frac{(A / 2+\eta) e^{(A / 2+\eta) y}-(A / 2-\eta) e^{(A / 2-\eta) y-2 \eta}}{e^{A / 2} \lambda^{-\lambda^{2}\left(e^{\eta}-e^{-3 \eta}\right)}}\right\} \\
{\left[\frac{\partial u}{\partial y}\right]_{y=0}=\tau=\left[\frac{e^{-\lambda^{2} t}\left[\left(\frac{A}{2}+\eta\right)-\left(\frac{A}{2}-\eta\right) e^{-2 \eta}\right]}{e^{\frac{A}{2}-\lambda^{2}}\left(e^{\eta}-e^{-3 \eta}\right)}\right] }
\end{aligned}
$$

\section{Nulsselt Number}

From (22),temperature gradient is given as:

$\left[\frac{\partial \theta}{\partial y}\right]=\frac{\lambda e^{A(1-t)}}{2 \sin \lambda \cos \lambda}(\cos \lambda \cos \lambda y-\sin \lambda \sin \lambda y)$

$\mathrm{Nu}=-\left[\frac{\partial \theta}{\partial y}\right]=\frac{\lambda e^{A(1-t)}}{2 \sin \lambda \cos \lambda}(\sin \lambda \sin \lambda y-\cos \lambda \cos \lambda y)(25)$

$\mathrm{Nu}$ at $\mathrm{y}=-1, \mathrm{y}=0$ and $\mathrm{y}=+1$ are

$\mathrm{Nu}=-\left[\frac{\partial \theta}{\partial y}\right]_{y=-1}=\frac{\lambda e^{A(1-t)}}{2 \sin \lambda \cos \lambda}\left(2 \cos ^{2} \lambda-1\right)(26)$

$\mathrm{Nu}=-\left[\frac{\partial \theta}{\partial y}\right]_{y=0}=-\frac{\lambda e^{A(1-t)}}{2 \sin \lambda}(27)$
$\mathrm{Nu}=-\left[\frac{\partial \theta}{\partial y}\right]_{y=+1}=\frac{\lambda e^{A(1-t)}}{2 \sin \lambda \cos \lambda}\left(2 \sin ^{2} \lambda-1\right)(28)$

\section{Discussion of Result}

The effect of inclinedHartmann in an unsteady MHD Poiseuille flow between two infinite parallel porous plates with heat transfer were obtained. The velocity profile, and skin friction profile are shown graphically against $y$ for various values of the Hartmann $\mathrm{Ha}$, while, the temperature profile was plotted againsty for various Prandlt number.

Figures 1- 6 depict decrease in velocity as $\mathrm{Ha}$ increases with effect of increase in the angle of inclination on velocity. This is an indication of positive effect of the magnetic component retarding the velocity of the flow.

Figure 7shows that temperature decrease as a result of increase in PrandltnumberPr.If there is an increase in the Prandlt number shows a reduction in thermal conductivity and thus produces an increase in the coefficient of dynamic viscosity and specific heat at a constant pressure.

Table1 shows increase in Nuas time t increases and absolute reduction when the Prandlt number increases. While, Tables 2 and 3shows reductionin skin friction asHaincreases when both plates are non-porous. This gives an indication of the contribution of magnetic field to a decrease in velocity.

Tables 4 and 5 shows decrease in skin friction as time $t$ increases under various $\mathrm{Ha}$ number when both plates are porous and when the inclination angle increases.

\section{Summary and Conclusion}

It has been observed that high Hartmann number decreases the velocity as well as the skin friction. When the magneticfield is high it reducesthe energy loss through the plates. But large Nulsset number corresponds to more active convection. Also, when the Prandlt number increases it brought about a decrease in the temperature profile. Thus, it shows a significant contribution of magnetic field to the flow of an unsteady MHD Poiseuille flow between two infinite parallel plates in an inclined field with heat transfer.

This work has the potential applications in electric power generator, which shows that there will not be heat loss through the medium of the generation at various times, thus, energy generated will be maintained throughout the flow field. Also, at the extrusive of plastics in the manufacturing of Rayon and Nylon this has a significant contribution. 


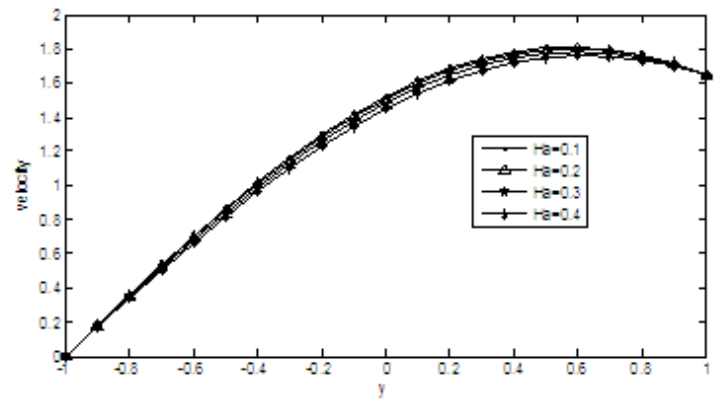

Figure 1: Velocity distribution for various Hartmann numbers $\left(\left[=15^{\circ}\right)\right.$ when both plates are non porous.

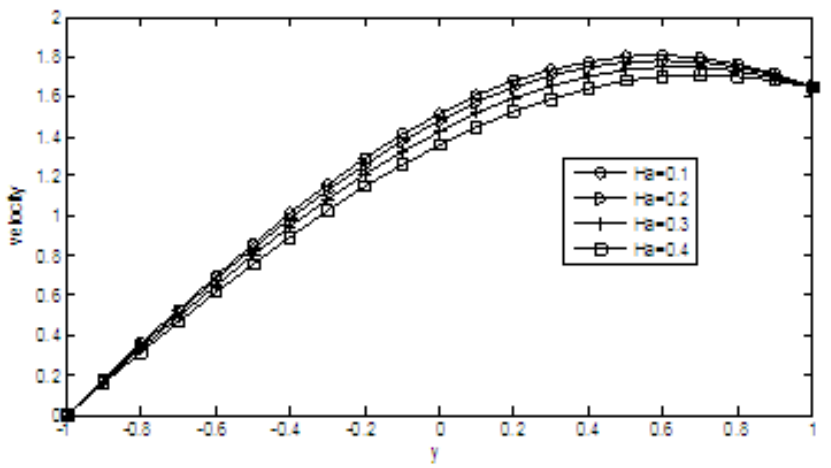

Figure2: Vebcity distribution for various Hartmann numbers $\left(\square=30^{0}\right)$ when both plates are non porous.

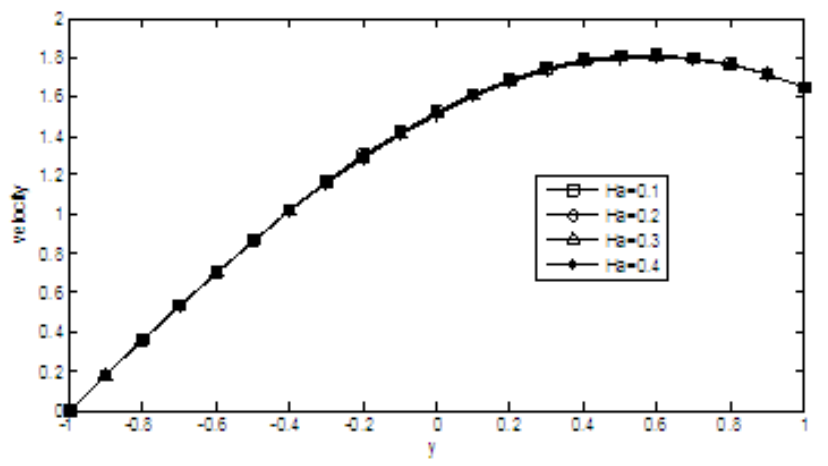

Figure 3: Vebcity distribution for various Hartmann numbers $\left(\square=60^{\circ}\right)$ when both plates are non porous.

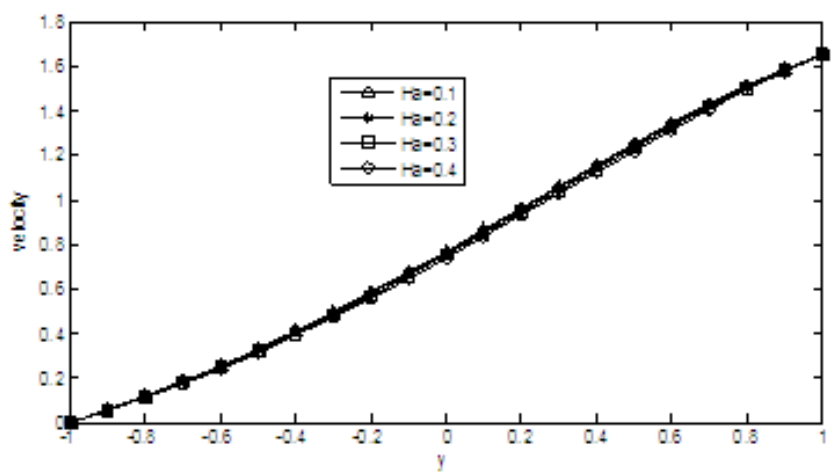

Figure4: Velocity distribution for various Hartmann number $\left(\square=15^{0}\right)$ when the lower plate is porous. 


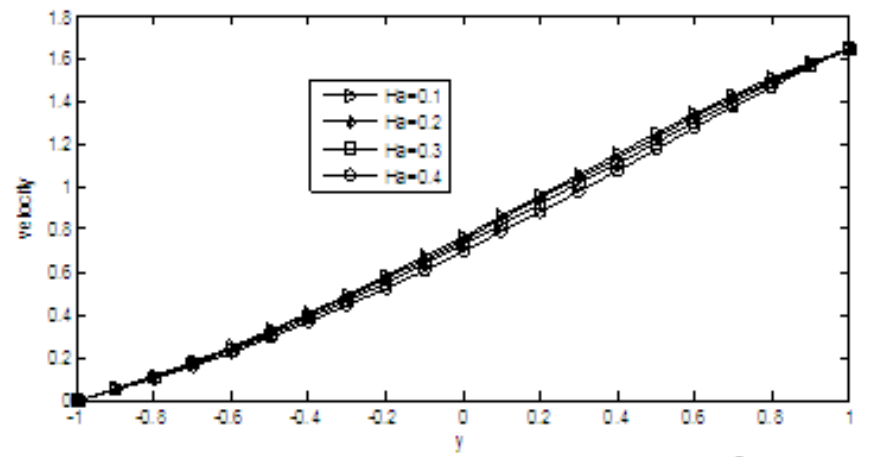

Figure5: Velocity distribution for various Hartmann Number $\left(\mathrm{I}=30^{\circ}\right)$ when the lower plate is porous.

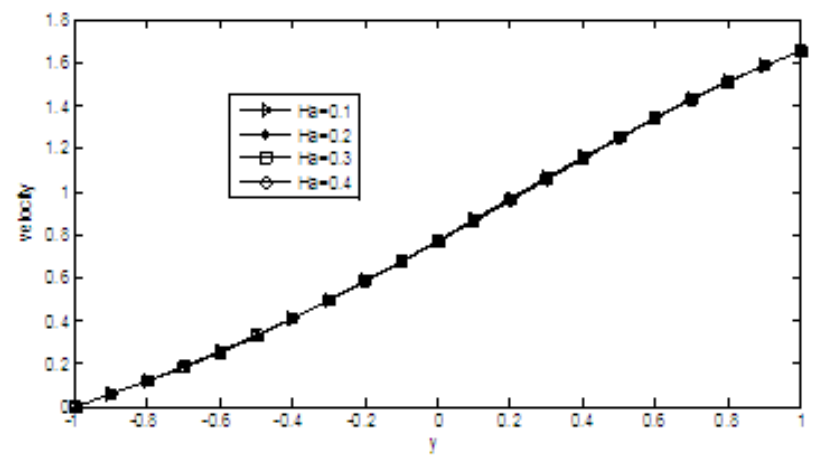

Figure6: Velocity distribution for various Hartmann Number $\left(\square=60^{\circ}\right)$ when the lower plate is porous

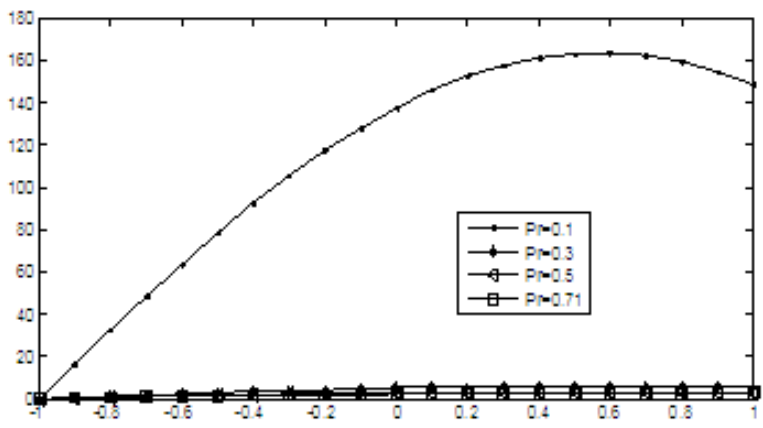

Figure 7: Temperature distribution for various $\operatorname{Pr}$ number.

Table 1: Variation of Nulsset numberNuwith different values of Pr andt.

\begin{tabular}{|l|l|l|l|l|}
\hline & $\mathrm{Pr}=0.1$ & $\mathrm{Pr}=0.3$ & $\mathrm{Pr}=0.5$ & $\mathrm{Pr}=0.71$ \\
$\mathrm{~T}$ & $\mathrm{Nu}$ & $\mathrm{Nu}$ & $\mathrm{Nu}$ & $\mathrm{Nu}$ \\
\hline 0.0 & $-1.3088 \mathrm{e}+004$ & -16.6563 & -4.3906 & -2.4300 \\
\hline 0.5 & -88.1867 & -3.1460 & -1.6152 & -1.2016 \\
\hline 1.0 & -0.5942 & -0.5942 & -0.5942 & -0.5942 \\
\hline 1.5 & -0.0040 & -0.1122 & -0.2186 & -0.2938 \\
\hline
\end{tabular}

Table 2: Variation of skin friction for various Hartmann number $\left(\alpha=15^{0}\right)$ when plates are non porous.

\begin{tabular}{|l|l|l|l|l|}
\hline $\mathrm{T}$ & $(\mathrm{Ha}=0.1)$ & $(\mathrm{Ha}=0.2)$ & $(\mathrm{Ha}=0.3)$ & $(\mathrm{Ha}=0.4)$ \\
& \multicolumn{1}{|c|}{$\tau$} & $\tau$ & $\tau$ & $\tau$ \\
\hline 0.0 & 1.6140 & 1.6103 & 1.6042 & 1.5958 \\
\hline 0.5 & 0.9789 & 0.9767 & 0.9730 & 0.9679 \\
\hline 1.0 & 0.5937 & 0.5924 & 0.5902 & 0.5871 \\
\hline 1.5 & 0.3601 & 0.3593 & 0.3580 & 0.3561 \\
2.0 & 0.2184 & 0.2179 & 0.2171 & 0.2160 \\
\hline
\end{tabular}


Unsteady Mhd Poiseuille Flow Between Two Infinite Parallel Plate In An Inclined Magnetic....

Table 3: Variation of skin friction for various Hartmann numbers $(\alpha=30)$, when plates are non porous

\begin{tabular}{|l|l|l|l|l|}
\hline $\mathrm{T}$ & $\mathrm{Ha}=0.1$ & $\mathrm{Ha}=0.2$ & $\mathrm{Ha}=0.3$ & $\mathrm{Ha}=0.4$ \\
& $\tau$ & $\tau$ & $\tau$ & $\tau$ \\
\hline 0.0 & 1.6124 & 1.6040 & 1.5901 & 1.5709 \\
\hline 0.5 & 0.9780 & 0.9729 & 0.9644 & 0.9528 \\
\hline 1.0 & 0.5932 & 0.5901 & 0.5850 & 0.5779 \\
\hline 1.5 & 0.3598 & 0.3579 & 0.3548 & 0.3505 \\
\hline 2.0 & 0.2182 & 0.2171 & 0.2152 & 0.2126 \\
\hline
\end{tabular}

Table 4: Variation of skin friction of the medium when lower plate is porous with various $\mathrm{Ha}, \alpha=15^{0}$.

\begin{tabular}{|l|l|l|l|l|}
\hline $\mathrm{T}$ & $\mathrm{Ha}=0.1$ & $\mathrm{Ha}=0.2$ & $\mathrm{Ha}=0.3$ & $\mathrm{Ha}=0.4$ \\
& \multicolumn{1}{|c|}{$\tau$} & $\tau$ & $\tau$ & $\tau$ \\
\hline 0.0 & 1.5709 & 1.5634 & 1.5511 & 1.5343 \\
\hline 0.5 & 0.9528 & 0.9483 & 0.9408 & 0.9306 \\
\hline 1.0 & 0.5779 & 0.5751 & 0.5706 & 0.5644 \\
\hline 1.5 & 0.3505 & 0.3488 & 0.3461 & 0.3423 \\
\hline 2.0 & 0.2126 & 0.2116 & 0.2099 & 0.2076 \\
\hline
\end{tabular}

Table 5:Variationof skin friction of the medium when lower plate is porous with various $\mathrm{Ha}, \alpha=30^{\circ}$

\begin{tabular}{|l|l|l|c|c|}
\hline $\mathrm{T}$ & $\mathrm{Ha}=0.1$ & $\mathrm{Ha}=0.2$ & $\mathrm{Ha}=0.3$ & $\mathrm{Ha}=0.4$ \\
& \multicolumn{1}{|c|}{$\tau$} & $\tau$ & $\tau$ & $\tau$ \\
\hline 0.0 & 1.5676 & 1.5505 & 1.5230 & 1.4864 \\
\hline 0.5 & 0.9508 & 0.9404 & 0.9238 & 0.9016 \\
\hline 1.0 & 0.5767 & 0.5704 & 0.5603 & 0.5468 \\
\hline 1.5 & 0.3498 & 0.3460 & 0.3398 & 0.3317 \\
\hline 2.0 & 0.2122 & 0.2098 & 0.2061 & 0.2012 \\
\hline
\end{tabular}

\section{References}

[1]. A.K. Al-Hadhrami, L. Elliot, M.D.Ingham, X.Wen,(2003): Flow through horizontal channels of porous materials, Inter. J. of Energy Research 27, 875-889.Raptis, C. Perkidis, (1999): Radiation and Free Convention flow past a moving plate, Int, J. of Applied Mechanics and Engineering, 4(4):817-821.

[2]. Gbadeyan, J.A. Idowu, A.S., Areo, A.O. and Olaleye. O.P.(2010):The radiative effect on velocity, magnetic and temperature fields of a magneto hydrodynamic oscillatory flow past a limiting surface with variable suction. Journal of Mathematical Sciences.21 (4), 395-411

[3]. C.B. Singh,(1993): MHD Steady flow of liquid between two parallel plates, In: Proc. of First Conference of Kenya Mathematical Society, 24-26.

[4]. C.B.Singh, P.c. Ram,(1978): Unsteady MHD Fluid Flow through a channel: Journal of Scientific Research, 28(2).

[5]. D.G.Drake, (1965): Flow in a channel due to a periodic pressure gradient, Quart. Journal of Mech. And Appl. Maths., 18(1).

[6]. E.M.Sparrow, R.D.Cess,(1961): The effect of a magnetic field on free convention heat transfer, Int. J. heat Mass Tran., 3(4): 267274.

[7]. H. Alfven,(1942): Existence of electromagnetic-hydrodynamics waves, Nature, 150(3805), 405-406.

[8]. J.A. Shercliff, (1956):Entry of conducting and non-conducting fluids in pipes, Journal of Mathematical Proc. of the Cambridge Philosophical Soc., 52, 573-583.

[9]. John Mooney, Nick Stokes,(1997): Time-varying MHD flows with free surfaces, Inter. Conf. on CFD in Mineral Metal Processing and Power Generation CSIRO.

[10]. P.A.Davidson, (2001): An introduction to MHD, First Edition, Cambrige University Press, UK.

[11]. P.C. Ram, C.B. Singh, U. Singh, (1984): Hall effects on Heat and Mass Transfer flow through porous medium, Astrophysics Space Science, 100: 45-51.

[12]. S. Ganesh, S.Krishnambal, (2007): Unsteady MHD Stokes flow of viscous fluid between two parallel porousplates, Journal of Applied Sciences, 7, 374-379.

[13]. Stamenkovic M. Zivojin, Dragiza D. Nikodijevic, Bratislav D. Blagojevic, Slobodan R. Savic, (2010): MHD flow and Heat Transfer of two immiscible fluids between moving plates, Transaction of the Canadian Society for Mechanical Engineering, 4(34): 351-372.

[14]. U.S. Rajput, P.K. Sahu, (2011): Transient free convection MHD flow between two long vertical parallel plates with constant Temperature and variable mass diffusion, Int. Journal of Math. Analysis, 34(5): 1665-1671.

[15]. V.M.Soundalgekar, Mohammed Abdulla-Ali,(1986): Free Convention effects on MHD flow past an impulsively started infinite vertical isothermal plate, Reg. J. Energy Heat and Mass Transfer 8(2): 119-125.

[16]. W.A. Manyonge, D.W. Kiema, C.C.W. Iyaya, (2012): Steady MHD Poiseuille Flow between two infinite parallel porous plates in an inclined magnetic field, Inter. Journal of Pure and Applied Maths., 76(5): 661-668. 